\title{
An Arc Discharge by Closely Situated Electrodes for Synthesis of Nanostructures
}

\author{
Nikola Koprinarov $^{1, ~ a, ~ M i k o ~ M a r i n o v ~}{ }^{2, ~ b}$, Mariana Konstantinova ${ }^{1, c}$ \\ ${ }^{1}$ Central Laboratory for Solar Energy and New Energy Sources, Bulgarian Academy of \\ Sciences, 72, Tzarigradsko chaussee Bulv., 1784 Sofia, Bulgaria \\ ${ }^{2}$ Institute of Physical Chemistry, Bulgarian Academy of Sciences, bl. 11 Acad. G. Bontchev \\ Str., 1113 Sofia, Bulgaria

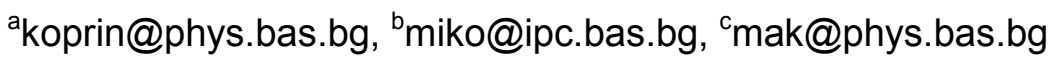

Keywords: Arc discharge, nanostructures, Si nanowires, ZnO nanowires, Si whiskers.

\begin{abstract}
Geometry of electrodes, distance between them, work atmosphere and ambient temperature are the important factors, which determine quantity and variety of structures synthesized via arc discharge. Usually, electrodes of different cross-section are placed away from each other, allowing a large vapor stream directed into the reactor inside to be obtained. Generally, the anode is thinner than the cathode; it heats up to a high temperature, sublimates and supplies the carbon vapor required for nanoparticle synthesis. In contrast to this commonly used approach, when electrode dimensions are appropriately chosen and electrodes placed closely together, temperature interaction between them becomes considerable, discharge area constrains and hot electrodes can be used as heaters for the evaporation of materials of high melting point.
\end{abstract}

\section{Introduction}

Arc discharge was one of the first methods used for carbon nanostructure synthesis $[1,2,3,4,5]$. It showed high productivity and the synthesized structures possessed excellent quality. Due to that the method is still widely employed. Carbon vapor required for this process was obtained by sublimation of the anode heated up to a high temperature and the energy came from the arc. Rarely, when catalyst was used for carbon nanostructure synthesis the catalyst could be incorporated in the carbon electrodes and, as a result, simultaneously evaporated with carbon. But evaporation of a different from carbon material, only, via arc between the carbon electrodes has not been realized up to now.

Our investigations showed that in contrast to the commonly used approach, when the two electrodes were placed closely together, the temperature interaction between them became considerable and the discharge area constrained. That allowed electrode temperature to be managed via appropriate choice of electrode dimensions and the sublimation velocity of electrodes to be strongly reduced. The aim of the investigations described here was to reduce carbon electrode evaporation and to use high electrode temperature for the evaporation of materials of high melting point in an appropriate manner.

\section{Experiment}

A sketch of the apparatus used for arc discharge by closely situated electrodes is shown in Fig. 1. The electrodes were made of pure carbon (99.999). The cathode had two openings filled up with the material intended for evaporation. The electrode ends, connected to a current source, were water cooled. The arc discharge took place in a stainless steel container, which was evacuated to high vacuum and afterwards filled in with $\mathrm{Ar}$ to a pressure of $3 \times 10^{4} \mathrm{~Pa}$. Despite of the low electrode consumption, if such emerged as a result of anode sublimation, the distance between the electrodes was kept at $0.5 \mathrm{~mm}$ by continuously moving the anode. So, due to the permanent distance control, the conditions of the arc discharge remained constant. 


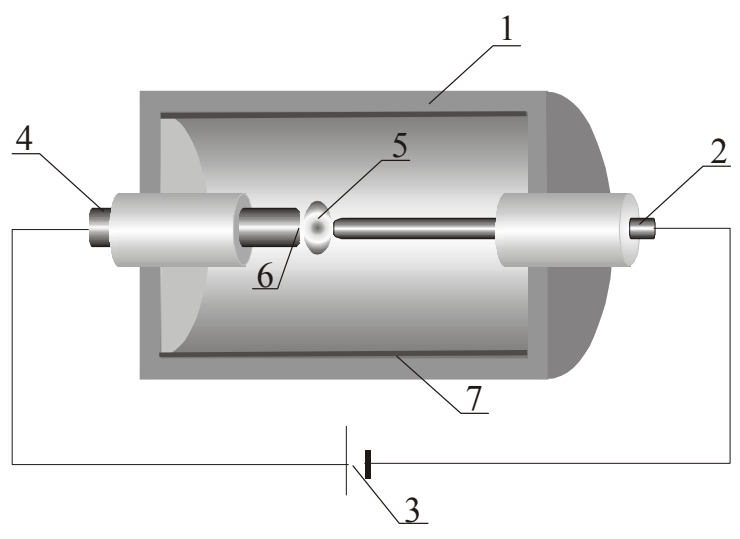

a
1 chamber

2 cathode

3 DC power supply

4 anode

5 ARC

6 deposit

7 soot

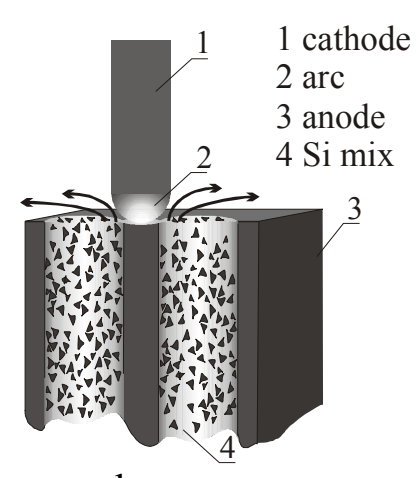

b

Fig. $1 \mathrm{a}$ - Scheme of the arc discharge apparatus and b- the anode cross-section.

\section{Results and discussion}

In the method developed, the temperatures of the two electrodes were equalized by cooling the anode and heating the cathode. For this purpose water-cooling was used and the anode cross-section was made bigger for to reduce its temperature and to stop its sublimation. In contrast, the small cathode diameter increased its temperature and its thermo electron emission grew which became sufficient to support stable arc burning. After ignition, the arc was situated between the cathode and that part of the anode that was between the openings. Using a radiation thermometer it was estimated that the temperature in the arc zone was over $3000{ }^{\circ} \mathrm{C}$, decreasing to $1100{ }^{\circ} \mathrm{C}$ at the anode periphery. Because of temperature distribution in the opening, all Si particles might be expected to be melt during arc discharge. To separate the drops of molten $\mathrm{Si}$ in the opening and to evaporate only those Si drops that are near the arc, a mix of Si particles and carbon powder was used because it was well known that up to $2000{ }^{0} \mathrm{C}$ carbon did not react with $\mathrm{Si}$.

The material grown on the anode was studied by Scanning Electron Microscopy (SEM), with no additional treatment. We observed that the particles deposited on the openings edges and on the protruding places had the shapes shown in Fig. 2a. These whiskers had length of up to $20 \mu \mathrm{m}$ and diameter up to $3 \mu \mathrm{m}$. We also observed almost uniform Si nanowires beyond the whisker region, in the direction to the periphery (Fig. 2b). These nanowires had diameters from 0.03 to $0.3 \mu \mathrm{m}$ and lengths from 0.3 to $5 \mu \mathrm{m}$. Splitting of the growing Si crystals frequently occurred. Because of this, some of the nanowires changed their direction or branched out several times during their growth (Fig. 2c).

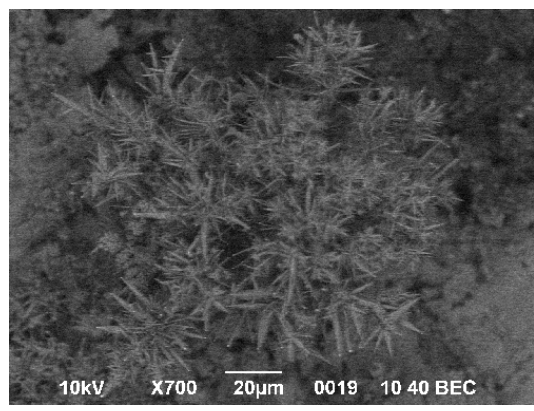

a

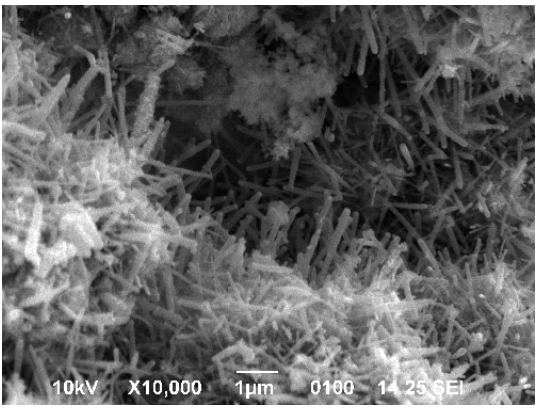

b

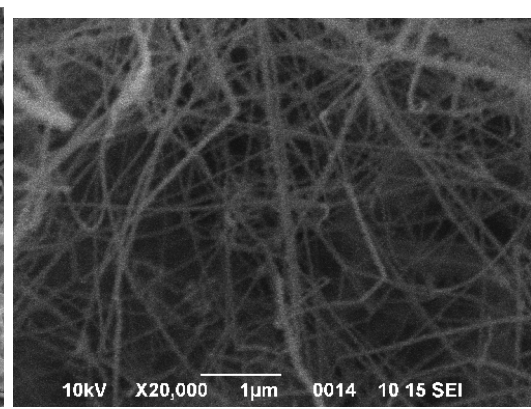

C

Fig. 2 Si sub-micron particles and nanostructures created on the anode: a- Si whiskers, b-Si nanowires and c-split Si nanowires

The proposed method was used for $\mathrm{ZnO}$ particle synthesis, also, starting from $\mathrm{ZnO} / \mathrm{C}$ mixture in the cathode openings. $\mathrm{ZnO}$ and carbon were powdered. Carbon could not react with $\mathrm{ZnO}$ and was used to prevent $\mathrm{ZnO}$ particle agglomeration and to homogenize the temperature into the openings. In difference to $\mathrm{ZnO}$ deposition methods known from the literature $[6,7,8,9]$ as techniques for $\mathrm{ZnO}$ nanowires synthesis, we have not used catalysts during the process, neither in the powder 
mixture nor deposited on Si wafer, used as a substrate. Fig. 3a shows a part of the substrate area obtained by our experiments and covered by a perfect column of $\mathrm{ZnO}$. The nanowires are perpendicular to the substrate surface. Energy Dispersive X-ray spectroscopy (EDX) estimated the $\mathrm{Zn} / \mathrm{O}$ fraction of atoms to be 55/45, which did not correspond to $\mathrm{ZnO}$ stoichiometry. On the basis of this, it was possible to suppose that $\mathrm{ZnO}$ has been partially decomposed during evaporation and $\mathrm{Zn}$ concentration on the top of the growing particles increased. The gradient in $\mathrm{Zn}$ concentration along the wires was confirmed thorough EDX spectroscopy. Probably, high $\mathrm{Zn}$ concentration on the particle top has caused a vapor liquid solid (VLS) deposition to some extent, and has resulted in well ordered nanowires. The diameter of nanowires was in the range of $200-300 \mathrm{~nm}$ and their length was several micrometers. The covering of large areas by homogenous structures was also valuable for their application as gas sensors and for medical purposes, due to their very large active surface.

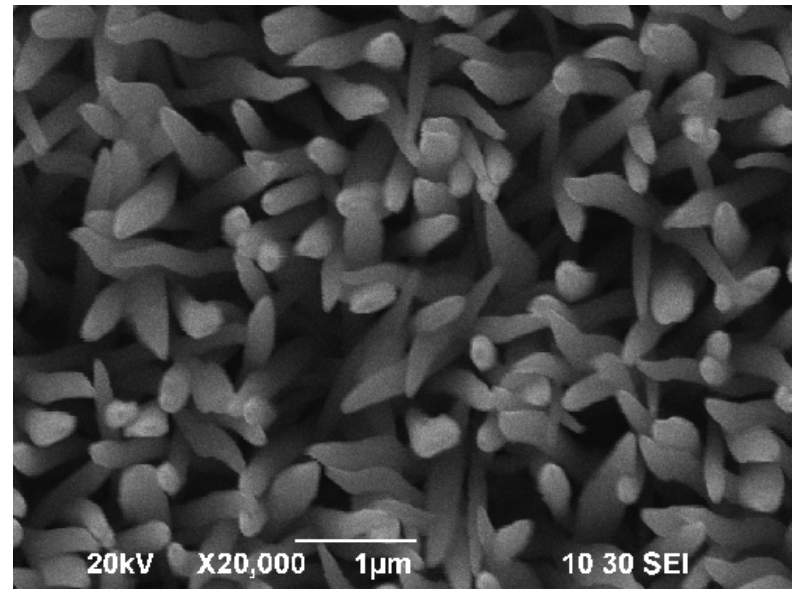

a

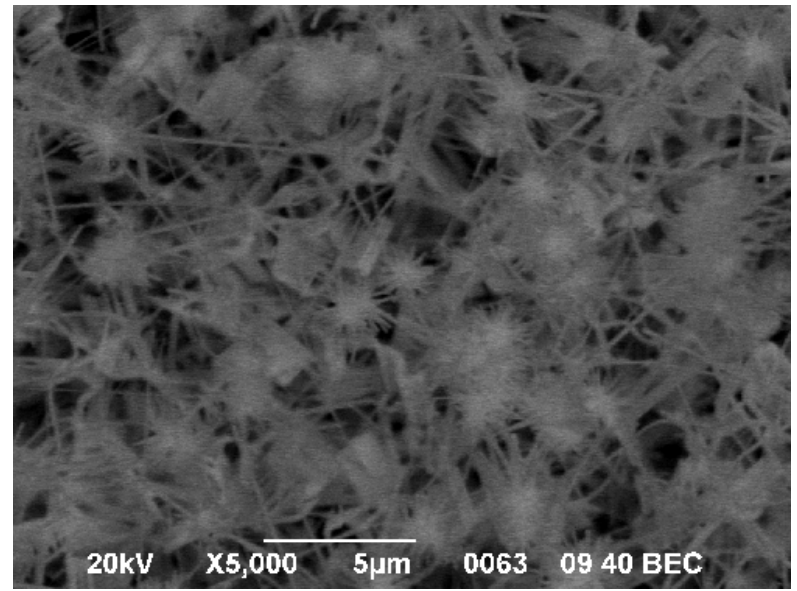

b

Fig. $3 \mathrm{ZnO}$ nanowires: a- nanowires perpendicular to the Si surface and b- free standing wires.

Due to the low $\mathrm{ZnO}$ chemical activity to the Si substrate, the thermal contact between the both media was strongly reduced. As a result, $\mathrm{ZnO}$ particles have grown as free standing wires at low substrate temperature. Those were up to $10 \mu \mathrm{m}$ long and with diameters of 50-150 nm (see Fig. 3b).

\section{Conclusions}

The obtained results show that electrode temperature can be successfully managed via an appropriate choice of electrode dimensions and kept lower than that necessary for carbon sublimation. That allows the heated electrodes to be employed as crucibles for $\mathrm{Si}$ and $\mathrm{ZnO}$ evaporation. The obtained sub-micron particles and nanostructures (whiskers, nanotubes and wires) have good quality on large substrate area.

The possibility for covering large areas by perfect SiNW and $\mathrm{ZnO}$ wires or tips is important for the use of such morphologies as field emitters of electrons, in gas sensors and for medical applications.

Acknowledgements. This work was funded by the Bulgarian National Scientific Fund under contract No 241/18.12.08 


\section{References}

[1]R. R. Schlittler, J. W. Seo, J. K. Gimzewski, C. Durkan, M. S. M. Saifullah and M. E. Welland: Science, Vol. 292 (2001), p.1136.

[2]M. Nishioa, S. Akitaa and Y. Nakayamaa: Thin Solid Films, Vol. 464-465 (2004), p.304.

[3]F. J. M. Rietmeijer, A. Rotundi and D. Heymann: Fullerenes, Nanotubes and Carbon Nanostructures, Vol. 12, No 3 (2004), p. 659.

[4]H. Kathyayinib, N. Nagarajua, A. Fonsecab and J. B. Nagy: Journal of Molecular Catalysis A: Chemical, Vol. 223 (2004), p. 129.

[5]Z. Yu, De Chen, B. Totdal, T. Zhao, Y. Dai, W. Yuan and A. Holmen: Applied Catalysis-A: General, Vol. 279 (2005), p. 223.

[6]S. Eustis, D. C.Meier, M. R. Beversluis and B. Nikoobakht: ACS Nano, Vol. 2, No 2 (2008), p. 368.

[7]M. H. Huang, Y. Wu, H. Feick, N. Tran, E. Weber and P. Yang: Adv. Mater., Vol. 13, No 2, (2001), p. 113.

[8]Ch.Y. Lee, T. Y. Tseng, S.Y. Li and P. L. Tamkang: Journal of Science and Engineering, Vol. 6, No 2 (2003), p. 127.

[9]Zh. L. Wang: J. Phys.: Condens. Matter, Vol. 16 (2004), R829. 